Received: 2018.09.17 Accepted: 2018.10.19 Published: 2019.02.07

Authors' Contribution: Study Design A Data Collection B Statistical Analysis C Data Interpretation D Manuscript Preparation E Literature Search F Funds Collection G

Corresponding Author: Conflict of interest:

BDE

E

\title{
Spontaneous Rectus Sheath Hematoma: An Uncommon Cause of Acute Abdominal Pain
}

Department of Internal Medicine, United Health Services Hospitals, Wilson Medical Center, Johnson City, NY, U.S.A.

\begin{abstract}
Patient: Female, $\mathbf{5 0}$
Final Diagnosis: Spontaneous rectus sheath hematoma

Symptoms: Cough $\bullet$ pelvic pain

Medication: -

Clinical Procedure: -

Specialty: General and Internal Medicine

Objective: Rare disease

Background: A clinical condition that is often misdiagnosed, rectus sheath hematoma (RSH) is usually seen in the context of blunt abdominal trauma and/or anticoagulation therapy, rarely occurring spontaneously. We present a case of spontaneous rectus sheath hematoma (SRSH) without obvious risk factors and review the literature regarding diagnosis modalities and management. The aim of this case presentation is to highlight this rare clinical condition and emphasize the role of the physical exam in determining the appropriate treatment approach.

Case Report: A 50-year-old woman presented to the emergency room with right-sided pelvic pain for one day. Her medical history was specifically notable for recent coughing due to acute bronchitis, as well as the use of NSAIDs. Physical examination revealed marked tenderness in the hypogastric and right lower quadrant, with guarding and fullness in the same area. Laboratory investigation showed mild anemia and normal coagulation tests. Computed tomography demonstrated a right rectus muscle hematoma measuring $8.5 \times 8.5 \mathrm{~cm}$ and extending into the lower abdomen and the extraperitoneal space, without active contrast extravasation. Close monitoring of vital signs and hemoglobin hematocrit levels along with supportive care with fluid resuscitation and pain control were initiated and the patient remained stable throughout her hospital stay.

Conclusions: $\quad$ Prompt recognition and management of SRSH are crucial. Physical examination is a key part of this process and imaging is the mainstay of diagnosis. Management remains for the most part supportive, although surgery or vascular embolization is required for uncontrolled hematomas with hemodynamic instability.
\end{abstract}

MeSH Keywords: Abdominal Pain $\quad$ Hematoma $\bullet$ Patient Care Management $\bullet$ Rectus Abdominis

Full-text PDF: $\quad$ https://www.amjcaserep.com/abstract/index/idArt/913246

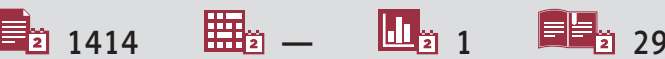




\section{Background}

A rare clinical condition that is often misdiagnosed, rectus sheath hematoma (RSH) is usually seen in the context of blunt abdominal trauma and/or anticoagulation therapy [1], rarely occurring spontaneously. The incidence seems to be increasing, possibly coinciding with expanding indications and use of anticoagulation. Often misdiagnosed, it accounts for $1.8 \%$ of acute abdominal pain syndromes based on a study of 1257 patients [2]. While usually considered a self-limiting condition, fatal cases were reported and mortality rates varied from $4 \%$ to $25 \%$ [3-5]. We present a case of spontaneous rectus sheath hematoma (SRSH) without obvious risk factors and discuss the literature regarding diagnosis modalities and management.

\section{Case Report}

A 50-year-old woman with a history of coronary artery disease status after LAD catheterization, hypothyroidism, dyslipidemia, depression, non-insulin dependent diabetes, obesity, and GERD presented with right-sided pelvic pain for one day. She described the $10 / 10$ pain as frank, continuous, and sharp. The pain started after a heavy bout of coughing, was located in the right groin and lower abdomen, prevented her from sleeping, was worsened by any movement or coughing, and was not alleviated by any factor. The pain was not associated with dizziness, loss of consciousness, nausea, or vomiting. The cough started the week prior and she was diagnosed with acute bronchitis, for which she received a course of azithromycin and prednisone. Both medications were given for 5 days, fully completed, and stopped 2 days prior to presentation. She also reported recent use of ibuprofen for pain. Long-term medications included aripiprazole $10 \mathrm{mg}$ daily, zolpidem $10 \mathrm{mg}$ nightly as needed, atorvastatin $20 \mathrm{mg}$ daily, carvedilol $3.125 \mathrm{mg}$ twice daily, escitalopram $20 \mathrm{mg}$ daily, levothyroxine $88 \mathrm{mcg}$ daily, metformin $500 \mathrm{mg}$ twice daily, and pantoprazole $40 \mathrm{mg}$ daily.

On physical exam, blood pressure was $103 / 58 \mathrm{mmHg}$, pulse rate was 64 beats/min, respiratory rate was 16 breaths/min, oxygen saturation was $97 \%$ on room air, and temperature was $98.4^{\circ} \mathrm{F}$. Her lungs were clear and cardiac auscultation revealed a normal S1 and S2 and absence of any murmur. There was marked tenderness in the hypogastric and right lower quadrant, with guarding. Palpation also revealed fullness of the same area. No ecchymosis was present. There was severe abdominal pain on bilateral straight leg raise in supine position, known as positive Carnett's sign. The right femoral and pedal pulses were faint and weak but present. The remainder of the physical examination was unremarkable.

Laboratory work-up showed hemoglobin $9.6 \mathrm{~g} / \mathrm{dL}$, hematocrit $31.5 \%$, platelets $114000 / \mu \mathrm{L}$, white blood count at $16.9 \mathrm{~K} / \mu \mathrm{L}$, INR 0.94, PT $10.6 \mathrm{~s}$, and APTT $25 \mathrm{~s}$. The remainder of the lab

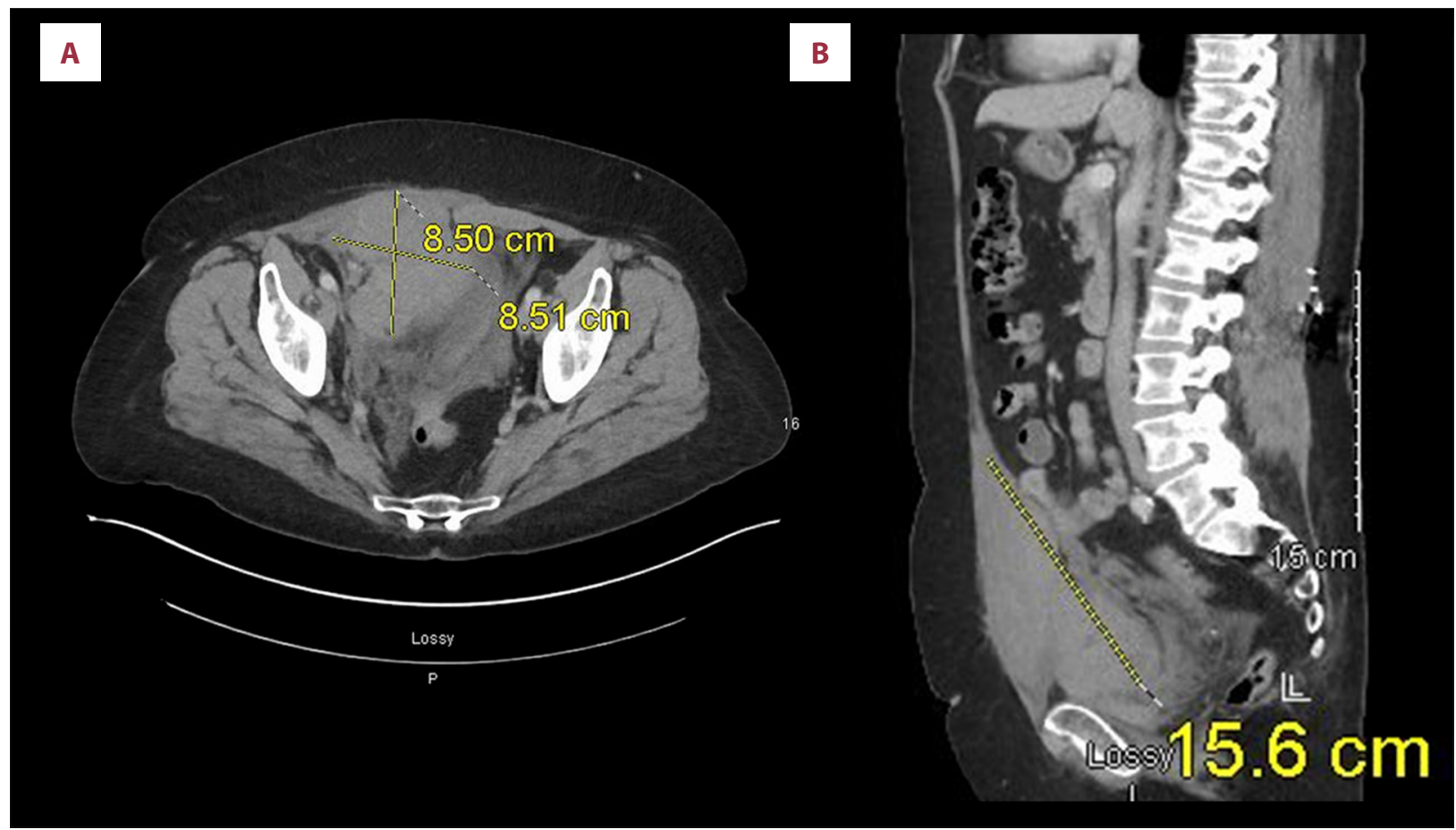

Figure 1. (A) Transverse CT section and (B) sagittal CT section of the pelvis. An intramuscular hemorrhage within the rectus muscle is seen inferiorly, extending into the extraperitoneal space of the right mid and mid lower aspect of the pelvis and measuring $15 \times 8.5 \times 8.5 \mathrm{~cm}$, with displacement of the superior bladder margin. 
work was within normal limits, with sodium $137 \mathrm{mmol} / \mathrm{L}$, potassium $3.6 \mathrm{mmol} / \mathrm{L}$, BUN $22 \mathrm{mg} / \mathrm{dL}$, creatinine $0.8 \mathrm{mg} / \mathrm{dL}$, albumin $3.7 \mathrm{~g} / \mathrm{dL}$, AST $23 \mathrm{IU} / \mathrm{L}$, ALT $21 \mathrm{IU} / \mathrm{L}$, alkaline phosphatase $59 \mathrm{IU} / \mathrm{L}$, and total bilirubin $0.5 \mathrm{mg} / \mathrm{dL}$. Computed tomography (CT) of the abdomen and pelvis with contrast showed a right rectus muscle hematoma measuring $8.5 \times 8.5$ $\mathrm{cm}$ (Figure 1A, 1B) and extending into the lower abdomen and the extraperitoneal space, without active contrast extravasation. Given the clinical suspicion for this finding and as CT was immediately available, CT was chosen over ultrasound for this patient given its high specificity as well as additional information provided including size, location, and possible extension of the hematoma. Close monitoring of vital signs and hemoglobin and hematocrit levels along with supportive care with fluid resuscitation and pain control were initiated and the patient remained stable throughout her one-day hospital stay. No surgical intervention was required.

\section{Discussion}

Rectus sheath hematoma (RSH) is usually seen in the context of blunt abdominal trauma and/or anticoagulation therapy [1], rarely occurring spontaneously. Despite being uncommon, its incidence seems to be increasing, possibly coinciding with expanding indications and use of anticoagulation. A vigorous and excessive contraction of the rectus abdominis [6] or direct abdominal trauma can lead to bleeding and hemorrhage secondary to rupture of one of the epigastric arteries and/or rectus abdominis muscle tear [7].

Hippocrates and Galen [6] were the first to describe RSH nearly 2500 years ago. The first case reported in the more recent literature was in 1857 by Richardson [8]. Often misdiagnosed, it accounts for $1.8 \%$ of acute abdominal pain syndromes based on a study of 1257 patients [2]. While usually considered a self-limiting condition, fatal cases have been reported and reported mortality rates vary from $4 \%$ to $25 \%$ [3-5].

Risk factors for the development of RSH are direct abdominal trauma $[2,5,9]$, iatrogenic damage after abdominal surgery $[6,10,11]$, use of anticoagulation $[6,12,13]$, coughing or intense abdominal wall muscles strain $[6,12,14,15]$ and pregnancy $[6,16]$. RSH is most common in women in their fifties $[9,12,17]$, and acute paroxysmal coughing is the most common triggering factor [5].

Abdominal pain is the most common symptom and is reported in $84-97 \%$ of cases. Other findings include an abdominal wall mass (63-92\%), positive abdominal guarding (49\%), nausea (23\%), and vomiting (15\%) $[1,6,9,12,18]$. Usually described as sharp, the abdominal pain is often non-radiating, continuous, and unilateral in the affected lower quadrant. As with our patient, the pain is usually exacerbated by movement.

An abdominal mass may be palpated and presents as tender and non-pulsating [19]. Carnett's sign is an increased tenderness after tensing the abdominal muscles (via lifting the head and shoulders or raising both legs with straight knees) and may help differentiate an intra-abdominal versus abdominal wall etiology [6], with a reported sensitivity of $81 \%$ and specificity of $88 \%$ [19]. Another valuable finding which suggests an intra-abdominally cause is Fothergill's sign, which is positive if the palpable mass does not cross the midline and does not change with flexion of the rectus muscles $[5,20]$. Ecchymosis was described by Teske in 1946 in $21 \%$ of RSH cases [9]. Peritoneal irritation signs and symptoms may be present, depending on the location and size of the hematoma. Hypotension and tachycardia may be present, depending on timing and degree of blood loss.

It is recommended to perform serial hemoglobin and hematocrit measurements for early detection of hypovolemia and to assess the need for blood transfusion. Importantly, however, the lack of hemoglobin and hematocrit drop alone does not rule out the presence of large hematomas [21,22]. Coagulation factors should be measured and can help determine the need for reversal in patients on anticoagulation therapy. However, $\mathrm{RSH}$ cases have also been described in patients with normal coagulation factor ranges $[21,23]$.

The use of ultrasonography (US) and computed tomography (CT) is the standard of care and has helped reduce the mortality and morbidity, as well as unnecessary surgical interventions [6]. US is very useful for both initial diagnosis and for monitoring RSH and is generally inexpensive, rapid, and readily available. When compared to US, CT scanning is reported to have a sensitivity and specificity approaching $100 \%$ and provides information such as the size, location, and extension of the hematoma. It is also very useful to rule out other abdominal pathologies [12,24]. CT aids in classification of RSH as developed by Berna and colleagues in 1996, with 3 types of RSH described with increasing severity. Type I RSH is small in size and confined within the rectus muscle without crossing the midline or dissecting fascial planes. Type II remains confined to the rectus muscle but can cross the midline or dissect the transversalis fascia plane. Type III is large, with evidence of hemoperitoneum and/or blood within the prevesical space (or cave of Retzius) [24]. Thus, diagnosis is established through a combination of supportive history and exam findings, along with confirmatory imaging.

The therapeutic options for RSH can be conservative or invasive [6]. Conservative management is more frequently chosen and is based on adequate analgesia, ice treatment, rest, and 
management of risk factors, and rarely reversal of anticoagulation [2,12]. Invasive therapy is considered in hemodynamically unstable patients with expanding hematoma, those not responding to medical resuscitation with IV fluids and/or blood transfusion, and in the presence of severe abdominal compartment or peritoneal irritation symptoms [12,24-26]. If invasive treatment is indicated, options are angioembolization of the bleeding vessel, imaging-guided drainage, and, very rarely, surgical evacuation, which carries a high morbidity risk [27-29].

\section{Conclusions}

SRSH is a potential medical emergency, particularly when atypical clinical presentations delay diagnosis and proper management. Early and accurate diagnosis of SRSH and its type are the most important factors to reduce morbidity and mortality, prevent unnecessary surgical intervention, and to implement and monitor conservative treatment when applicable. As our case demonstrates, clinicians should be mindful of this entity in the absence of classical risk factors.

\section{Conflicts of interest}

None.

16. Tolcher MC, Nitsche JF, Arendt KW, Rose CH: Spontaneous rectus sheath hematoma pregnancy: Case report and review of the literature. Obstet Gynecol Surv, 2010; 65;(8): 517-22

17. Costello J, Wright J: Rectus sheath haematoma: A diagnostic dilemma? Emerg Med J, 2005; 22: 523-24.

18. Moreno Gallego A, Aguayo JL, Flores B et al: Ultrasonography and computed tomography reduce unnecessary surgery in abdominal rectus sheath haematoma. Br J Surg, 1997; 84(9): 1295-97

19. Matsunaga S, Eguchi Y: Importance of a physical examination for efficient differential diagnosis of abdominal pain: diagnostic usefulness of Carnett's test in psychogenic abdominal pain. Intern Med, 2011; 50(3): 177-78

20. Aktürk O, Kayılıoğlu S, Aydoğan I et al: Spontaneous rectus sheath hematoma: An overview of 4-year single center experience. Indian J Surg, 2015 77(Suppl. 3): 1219-21

21. Zissin R, Gayer G, Kots E et al: Transcatheter arterial embolisation in anticoagulant-related haematoma - a current therapeutic option: A report of four patients and review of the literature. Int J Clin Pract, 2007; 61(8): 1321-27

22. Salemis NS, Gourgiotis S, Karalis G: Diagnostic evaluation and management of patients with rectus sheath hematoma. A retrospective study. Int J Surg, 2010; 8(4): 290-93

23. Hildreth $\mathrm{DH}$ : Anticoagulant therapy and rectus sheath hematoma. Am J Surg, 1972; 124(1): 80-86

24. Garcia Bear I, Macias Robles MD, Baldonedo Cernuda RF, Alvarez Perez JA: Spontaneous hematoma of the sheath of the rectus abdominis muscle: A challenge for the diagnosis. Emergencias, 2000; 12: 269-71

25. Yamada Y, Ogawa K, Shiomi E, Hayashi T: Images in cardiovascular medicine. Bilateral rectus sheath hematoma developing during anticoagulant therapy. Circulation, 2010; 121(15): 1778-79

26. Berna JD, Garcia-Medina V, Guirao J, Garcia-Medina J: Rectus sheath hematoma: diagnostic classification by CT. Abdom Imaging, 1996; 21(1): 62-64

27. Jafferbhoy SF, Rustum Q, Shiwani MH: Abdominal compartment syndrome - a fatal complication from a rectus sheath haematoma. BMJ Case Rep, 2012; 2012: pii: bcr1220115332

28. Toyonaga J, Tsuruya K, Masutani K et al: Hemorrhagic shock and obstructive uropathy due to a large rectus sheath hematoma in a patient on anticoagulant therapy. Intern Med, 2009; 48(24): 2119-22

29. Fitzgerald JE, Fitzgerald LA, Anderson FE, Acheson AG: The changing nature of rectus sheath haematoma: Case series and literature review. Int I Surg, 2009; 7(2): 150-54 